\title{
ALGUNAS ALTERACIONES ENZIMÁTICAS EN PAPA CAUSADAS POR FUNGICIDAS
}

\section{SOME ENZIMATIC ALTERATIONS IN POTATO CAUSED BY FUNGICIDES}

\author{
Rocío Serrano-Cervantes ${ }^{2}$, Héctor Lozoya-Saldaña ${ }^{1} *$ \\ María-Teresa B. Colinas y León ${ }^{1}$ y S. Gerardo Leyva-Mir ${ }^{2}$
}

\begin{abstract}
IInstituto de Horticultura, Departamento de Fitotecnia, ${ }^{2}$ Posgrado en Protección Vegetal, Departamento de Parasitología Agrícola, Universidad Autónoma Chapingo. Km. 38.5 Carretera México-Texcoco. 56230, Chapingo, Texcoco, Estado de México. Tel. 01-(595)-952-1500 exts. 6135 y 5224.
\end{abstract}

*Autor para correspondencia (picti87@gmail.com)

\section{RESUMEN}

El manejo químico de las plagas y enfermedades de la papa (Solanum tuberosum L.) es la estrategia fitosanitaria más práctica. Algunos fungicidas actúan directamente sobre la planta, generalmente en presencia del patógeno, sin especificar si el hospedante responde al patógeno, al agroquímico o a ambos. El presente estudio tuvo como objetivo evaluar algunas alteraciones enzimáticas de defensa de las plantas estimuladas por la aplicación de fungicidas sistémicos en ausencia de patógenos, e identificar posible especificidad de reacción enzimática a determinado producto. Bajo invernadero, se hicieron aspersiones foliares semanales, a media dosis y dosis completa, de fungicidas a plantas de papa de la variedad Ágata, y se colectó follaje con la misma frecuencia para evaluar la presencia y acción de varias enzimas relacionadas con reacciones de defensa no específica. La cantidad de fenoles totales (FEN) fue muy parecida entre tratamientos, excepto por la dosis completa de fluoxastrobina, que estimuló su síntesis por encima de los demás fungicidas al final del ciclo. La acción de la superóxido-dismutasa (SOD), por el contrario, se estimuló más durante la primera mitad del ciclo de crecimiento, sobre todo en el testigo (agua). Posteriormente se modificó su actividad a la baja, excepto otra vez por fluoxastrobina en media dosis, que mantuvo la actividad de la SOD por encima de la estimulación inducida por los otros fungicidas. En las dos dosis los tratamientos estimularon uniformemente la actividad de la peroxidasa (POX) por encima del testigo al final del ciclo. La actividad de la catalasa (CAT) disminuyó continuamente a lo largo del ciclo en las plantas testigo, mientras que los tratamientos estimularon su actividad mayormente a la mitad del ciclo, sin estímulo específico sobresaliente por algún fungicida en particular sobre esta enzima. Las correlaciones entre las enzimas POX-CAT, POX-SOD, FEN-SOD Y FEN-POX a los agroquímicos fueron significativas, lo que indica paralelismo o similitud de respuestas o de dependencia entre ellas a más de un producto, de reacciones no específicas, por el estímulo químico externo. Se identificó especificidad temprana de fosetil-Al como estimulante de POX, y de fluoxastrobina para SOD.

Palabras clave: Solanum tuberosum, enzimas de defensa, FEN, SOD, POX, CAT.

\section{SUMMARY}

Chemical pest management in potatoes (Solanum tuberosum L.) is the most practical approach. Some fungicides have direct action over the plant, usually in the presence of the pathogen, with no clarification on the host reaction either to the pathogen, to the product or to both. The objective of this study was to evaluate some plant defense enzymatic alterations stimulated by the application of systemic fungicides in the absence of pathogens, and to identify a possible specificity of an enzymatic reaction to a given product. Under greenhouse conditions, potato plants cv. Ágata were weekly sprayed at half and complete dosages of fungicides, sampling foliage with the same frequency followed by analyzing presence-action of several enzymes related to non-specific defense reactions. Total amount of phenols (FEN) was pretty close among treatments, except for the complete dosage of fluoxastrobin which stimulated phenol synthesis over the other fungicides at the end of the growing cycle. Superoxide dismutase (SOD) activity, on the other hand, was more stimulated along the first half of the growing cycle, especially in the controls. Its action was more uniform among treatments later on, except again for the half dosage of fluoxastrobin, which kept SOD activity higher than the rest of the fungicides. All treatments uniformly stimulated peroxidase (POX) activity over the control (water) at the end of the cycle. Catalase (CAT) activity continuously decreased along the growing cycle in the control plants, while the fungicide treatments stimulated its activity mainly at midseason, with no specific outstanding stimuli by any particular fungicide over this enzyme. Significant interactions were detected between POX and CAT, POX and SOD, and FEN with SOD and POX, as an indication of similar responses or mutual dependency among them to more than one product on non-specific reactions due to the external chemical stimuli. Early specificity was identified for fosetyl-Al as POX stimulant, and for fluoxastrobin for SOD. CAT

Index words: Solanum tuberosum, defense enzymes, FEN, SOD, POX

\section{INTRODUCCIÓN}

El cultivo de la papa (Solanum tuberosum L.), es uno de los que más agroquímicos utiliza entre las especies hortícolas comestibles cultivadas, ya que este control químico de sus plagas y enfermedades es la forma más práctica de su manejo fitosanitario. Existen evidencias sobre la acción de algunos fungicidas directamente sobre la planta, pero generalmente en presencia del patógeno (Juárez et al., 2010; Lozoya-Saldaña et al., 2007; Robledo et al., 2012) Una alternativa de manejo de enfermedades de plantas se basa en la "resistencia inducida", mediante la cual las plantas son protegidas de las enfermedades causadas por hongos, ya sea a través de una infección inicial por un patógeno (Kuúc, 1982) o por la aplicación de productos químicos sintéticos que activan respuestas de resistencia de la planta (Gottsein y Kuúc, 1989; Kessmann et al., 1994; White, 1979).

Para que ocurra la inducción de la defensa, es necesaria la mediación de sistemas de reconocimiento específico, mediante los cuales la planta reconoce la presencia del 
patógeno (Hutcheson, 1998). Después de la infección, las células infectadas en las variedades resistentes pierden rápidamente su turgencia, se necrosan y mueren; mientras que las de variedades susceptibles sobreviven más tiempo. Los procesos involucrados en la reacción hipersensible comprenden la pérdida de permeabilidad de las membranas celulares, aumento de la respiración, acumulación y oxidación de compuestos fenólicos y la producción de fitoalexinas (Agrios, 2007).

Algunos de los compuestos químicos del metabolismo vegetal más comúnmente asociados con reacciones de defensa de las plantas son los fenoles (FEN), la superóxido dismutasa (SOD), la peroxidasa (POX), y la catalasa (CAT), entre otros (Agrios, 2007; Hutcheson, 1998). En la planta los FEN aumentan su concentración de forma considerable tras la entrada de un patógeno para protegerse de éste, y se ha comprobado que su síntesis después de la infección es mucho mayor en variedades resistentes que en las susceptibles (Dai et al., 1995). Varios fenoles relacionados con la resistencia a las enfermedades se encuentran profusamente en las plantas ya sean sanas o enfermas, pero su síntesis o acumulación aumenta después de haberse producido la infección. Estos compuestos son fenoles "comunes".

Otros compuestos químicos, las fitoalexinas, no los producen las plantas sanas, excepto cuando son estimuladas por algún patógeno o por el daño de un agente químico o mecánico, y son específicos por especie (Agrios, 2007; Kuúk, 1995). Las SOD constituyen una de las defensas primarias de las células frente al agobio oxidativo. Catalizan la reacción de oxidación-reducción del radical superóxido a peróxido de hidrógeno y posteriormente a oxígeno libre y agua Se localizan en la matriz mitocondrial, peroxisomas, en el citosol y en el estroma cloroplástico (Bowler et al., 1994). Las condiciones adversas como la sequía, bajas temperaturas y ataque por patógenos se relacionan con mayor actividad de la SOD (Monk et al., 1989).

La POX oxida a los compuestos fenólicos y aumenta la velocidad de su polimerización en sustancias similares a la lignina, que se depositan en las paredes y papilas celulares e interfieren con el posterior crecimiento y desarrollo de patógenos (McInnis et al., 2006). La CAT, al igual que la SOD, es una enzima antioxidante que cataliza la desintegración del peróxido de hidrógeno en agua y oxígeno, pero además se asocia con la muerte celular como respuesta de hipersensibilidad (Levine et al., 1994).

Existe evidencia de la inducción de resistencia a enfermedades con la aplicación de productos dirigidos o no al patógeno (Deckers y Creasy, 1989; Gottsein y Kuúc, 1989; Kessman et al., 1994; White, 1979). En el grupo donde se desarrolló la presente investigación también se ha trabajado con la presencia del patógeno o con la planta ya enferma (Lozoya-Saldaña et al., 2006; Lozoya-Saldaña et al., 2007; Robledo et al., 2012), y no queda claro si la resistencia inducida por los agroquímicos en el hospedante es consecuencia exclusivamente por la presencia del producto químico o por la interacción agroquímico-patógeno sobre la planta.

El grupo de Beltrán-García (Beltrán-García et al., 2006) menciona el efecto de una respuesta antioxidante de los fungicidas que interrumpen el flujo de electrones en la mitocondria, que puede conducir a la muerte celular mediante el aumento del $\mathrm{H}_{2} \mathrm{O}_{2}$, aunque sus estudios están más enfocados a las catalasas de los hongos. Entre los fungicidas de este tipo (interferencia con la respiración) se encuentran boscalid, carboxin, flutolanil, azoxistrobina, picoxistrobina, y fluoxastrobina, enfocados en gran medida a las enfermedades fúngicas del suelo.

Bajo esta consideración, en el presente estudio se seleccionaron fungicidas con diferentes modos de acción sobre el patógeno; a) fluoxastrobina, como inhibidor de la respiración, b) metalaxil, inhibidor de la síntesis de ácidos nucléicos, c) fosetil-Al, multisitio, alterador de la permeabilidad de las membranas, y d) mandipropamid, como inhibidor de la síntesis de pared celular (Copping y Hewitt, 1998; Kuck et al., 2011). Estos compuestos se aplicaron mediante aspersión foliar, con el objetivo de evaluar algunas reacciones de defensa de la planta de papa, en ausencia del patógeno, por exposición a dichos agroquímicos, y para dar seguimiento a posibles correlaciones o similitudes o diferencias de respuesta entre las enzimas a los fungicidas y así identificar posible especificidad de reacción enzimática a determinado producto.

\section{MATERIALES Y MÉTODOS}

Bajo invernadero se sembró el cultivar de papa Ágata en bolsas de plástico, en un diseño experimental completamente al azar. El experimento constó de aplicaciones semanales al follaje de dosis completa o media de cuatro fungicidas (ocho tratamientos) más el testigo con aspersión de agua, para un total de nueve tratamientos (Cuadro 1). Se inició la aspersión foliar de los productos a los $30 \mathrm{~d}$ después de la emergencia y hasta cuando la planta estuvo en etapa de iniciación de tubérculos, cinco semanas después. Cada semana se tomaron muestras de follaje antes de la aspersión (30 g de hoja de la parte media de la planta) para el posterior análisis enzimático.

\section{Obtención de extractos}

Polvo de acetona. Se obtuvo a partir de $20 \mathrm{~g}$ de hojas + 
$50 \mathrm{~mL}$ de acetona $100 \%\left(4^{\circ} \mathrm{C}\right)$ maceradas en licuadora y filtradas al vacío. Este proceso se repitió dos veces más con el mismo tejido, conservando el sobrenadante para el análisis de fenoles, y dejando secar el tejido a temperatura ambiente $\left(18^{\circ} \mathrm{C}\right)$. Tanto al sobrenadante como al tejido se les almacenó a $-4{ }^{\circ} \mathrm{C}$ hasta su posterior utilización en los ensayos (Alia-Tejacal et al., 2002; Martínez-Téllez y Lafuente, 1997).

Fenoles. A $0.025 \mathrm{~mL}$ del sobrenadante se agregaron 7.975 $\mathrm{mL}$ de agua desionizada $+0.5 \mathrm{~mL}$ del reactivo para fenoles de Folin-Ciocalteu (Sigma-Aldrich Data sheet 47641) + 1.5 $\mathrm{mL}$ de carbonato de sodio $20 \%$. La mezcla se agitó y dejó reposar $2 \mathrm{~h}$ en la oscuridad para posteriormente leer la absorbancia a $760 \mathrm{~nm}$ en un espectrofotómetro (Genesys 10 Thermo Scientific $\AA$, EUA). La cuantificación se realizó mediante una curva patrón de ácido tánico y la concentración de fenoles totales se registró como $\mathrm{mg} \mathrm{g}^{-1}$ p.f. (Alia-Tejacal et al., 2002; Cornide et al., 1994; Martinez-Tellez y Lafuente, 1997; Waterman y Mole, 1994).

Superóxido dismutasa (1.15.1.1. SOD). El método utilizado es el descrito por Beyer y Fridovich (1978). A $0.05 \mathrm{~g}$ de polvo de acetona en tubos de fondo plano, se agregó $5 \mathrm{~mL}$ de solución amortiguador fosfato $0.01 \mathrm{M} \mathrm{pH} 7.8$ frío, se homogenizó inmediatamente en hielo, se decantó en tubos para centrifuga, se centrifugó a 22,500 Xg por 20 min a $4{ }^{\circ} \mathrm{C}$ (Sorbal RC-5C $\AA$, USA) y se conservó a temperatura ambiente. En ausencia de luz se tomaron $3 \mathrm{~mL}$ de solución amortiguadora EDTA-metionina, NBT y triton, que se colocaron en tubo de ensayo de rosca. Se agregaron $0.5 \mathrm{~mL}$ de sobrenadante, se agitó, se adicionó rivoflavina, se volvió a agitar, se iluminó por 7 min con lámpara de luz fluorescente y se leyó la absorbancia a 560 nm (Genesys 10 Thermo Scientific $\AA$, USA). La actividad enzimática se reportó en unidades por gramo de peso fresco $\left(U g^{-1}\right.$ p. f. $)$.

Peroxidasa (EC.1.11.1.7, POX). La enzima se extrajo a partir de $0.05 \mathrm{~g}$ de polvo de acetona con $5 \mathrm{~mL}$ de Tris-HCl frio (pH 7.1) que contenía $1 \%$ de polivinilpirrolidona. Se mezcló en un homogeneizador de tejidos durante 50 s y la mezcla se centrifugó por $20 \mathrm{~min}$ a $22,500 \mathrm{Xg}$ a $4^{\circ} \mathrm{C}$ (Alia-Tejacal et al., 2002). El sobrenadante se utilizó para el ensayo de acuerdo con Flurkey y Jen (1978), con las siguientes modi- ficaciones: se hizo en un volumen total de $3 \mathrm{~mL}$, con $2.6 \mathrm{~mL}$ de amortiguador Tris- $\mathrm{HCl}(\mathrm{pH} 7.1), 0.25 \mathrm{~mL}$ de guayacol 0.1 M, $0.1 \mathrm{~mL}$ de peróxido de hidrógeno $0.25 \%$ y $0.05 \mathrm{~mL}$ del sobrenadante. El cambio de absorbancia se evaluó a los 30, 60, 120 y $180 \mathrm{~s}$ a 470 nm (mismo espectrofotómetro). La actividad enzimática se reportó como $\cup g^{-1}$ p. f., donde $\mathrm{U}=$ unidad de actividad enzimática, igual a la formación de $1 \mathrm{mmol} \mathrm{min}{ }^{-1}$ de tetraguayacol.

Catalasa (EC.1.11.1.6, CAT). La actividad enzimática de la CAT se determinó con la metodología descrita por AliaTejacal et al. (2002), Blackwell et al. (1990) y Lück, (1963). Se disolvieron $0.5 \mathrm{~g}$ del polvo de acetona con $5 \mathrm{~mL}$ de Tris- $\mathrm{HCl}$ frío, $\mathrm{pH}$ 8.5, con polivinilpirrolidona $1 \%$, en un homogeneizador de tejido. Se centrifugó a 12,500 Xg por 20 min a $4{ }^{\circ} \mathrm{C}$, y solo se conservó el sobrenadante (extracto crudo). Posteriormente se hizo una mezcla de $3 \mathrm{~mL}$ de 10 $\mathrm{mM}$ Tris- $\mathrm{HCl}(\mathrm{pH}$ 8.5) y $0.1 \mathrm{~mL}$ de peróxido de hidrógeno $0.88 \%$ en 100 mM de Tris- $\mathrm{HCl}$. Para iniciar la reacción se agregó $0.1 \mathrm{~mL}$ del sobrenadante y se cuantificó el cambio en absorbancia a $240 \mathrm{~nm}$ (mismo espectrofotómetro). La actividad enzimática se expresó en $U \mathrm{~g}^{-1}$ de peso fresco,

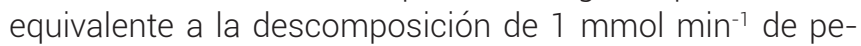
róxido de hidrógeno.

\section{Correlaciones}

Las variables consideradas para el presente estudio están muy relacionadas entre sí en sus rutas metabólicas, por lo que dado un mismo estímulo teóricamente no deben presentarse respuestas aisladas o independientes entre ellas (Lozoya-Saldaña et al., 2007). De modo que para entender los patrones de respuesta entre dos variables (enzimas), en este estudio se estimaron sus correlaciones con el método de máxima verosimilitud, mediante la obtención del coeficiente de correlación de Pearson (r).

\section{Análisis de resultados}

El análisis de los resultados se hizo mediante los programas SAS (Statistical Analysis System) y Excel Microsoft ${ }^{\circledR}$ mediante comparación de medias (unidades por gramo de peso fresco) y correlaciones entre las cuantificaciones de los comportamientos de las enzimas.

Cuadro 1. Fungicidas y dosis aplicadas semanalmente al follaje.

\begin{tabular}{|c|c|c|}
\hline Fungicida & Dosis completa/10 L de agua & Dosis media/10 L de agua \\
\hline Ridomil@ (Metalaxil-m) & $100 \mathrm{~mL}$ & $50 \mathrm{~mL}$ \\
\hline Aliette® (Fosetil-Al) & $25 \mathrm{~g}$ & $12.5 \mathrm{~g}$ \\
\hline Revus® (Mandipropamida) & $10 \mathrm{~mL}$ & $5 \mathrm{~mL}$ \\
\hline Vigold® (Fluoxastrobina) & $50 \mathrm{~mL}$ & $25 \mathrm{~mL}$ \\
\hline Testigo: agua & ---------------- & 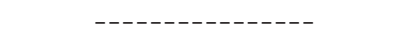 \\
\hline
\end{tabular}




\section{RESULTADOS Y DISCUSIÓN}

\section{Fenoles totales}

La estimulación en el contenido de fenoles totales fue muy parecida entre los productos aplicados y las dosis, donde el testigo (agua) se ubicó con valores intermedios a lo largo del ciclo (Cuadro 2) cuyo mayor incremento se presentó en la segunda fecha, para luego descender de modo uniforme. El máximo contenido de fenoles se presentó en el tercer muestreo, donde destaca el metalaxil a dosis completa y el fosetil-Al en media dosis, como los máximos estimulantes de fenoles totales. No obstante, al final del ciclo todos los productos fueron similares al testigo cuando se aplicaron en medias dosis. Se ha correlacionado la síntesis de fitoalexinas con la aplicación de fungicidas como el metalaxyl contra Phytophthora megasperma f. sp. Glycinea (Cahill y Ward, 1989) y el fosetil- Al con acumulación de resveratol en la vid (Deckers y Creasy, 1989). Estos fungicidas se utilizaron en el presente estudio, con máxima estimulación de fenoles totales a la mitad del ciclo.

\section{Superóxido dismutasa}

Se detectó un rápido incremento en la actividad de esta enzima en el segundo muestreo con respecto al primero al aplicar ambas dosis con todos los productos, incluido el testigo (Cuadro 2). No obstante, la SOD declinó gradualmente en el tratamiento con agua en los muestreos posteriores. Esta curva descendente también se observó con los tratamientos en ambas dosis. No obstante, destaca la estimulación de la SOD en el tercer muestreo por fosetil-Al y mandipropamid cuando se aplicaron en dosis completa, con descenso posterior pero con un ligero incremento de la actividad al final por la presencia de estos dos fungicidas. Es de notar la acción estimulante de la fluoxastrobina, en media dosis, que aunque también descendió su estímulo, mantuvo a la SOD por encima del resto de los productos en la tercera y quinta fecha.

El modo de acción del fosetil-Al sobre los oomicetos es multisitio, con alteración en la permeabilidad de las membranas, sin acción específica sobre las plantas, y del mandipropamid que es inhibidor de la síntesis de pared celular (Copping y Hewitt, 1998; Kuck et al., 2011), con lo que queda claro que no tienen relación con la actividad de la SOD (dismutación del superóxido), lo que explicaría que la planta no se defiende mediante mecanismos de depuración con la aplicación de estos productos, o con reacciones enzimáticas individuales, y que debería buscarse la respuesta en la interacción de actividades de otras enzimas en conjunto, relacionadas con las reacciones de defensa. La fluoxastrobina, por otro lado, inhibe respiración y se notó su acción estimulante sobre la SOD. Además, las moléculas consideradas como activadoras no deben ser tomadas como sustitutos de fungicidas, sino como una alternativa adicional dentro de una estrategia de manejo integrado (Lyon y Newton, 2000).

\section{Peroxidasa}

Los tratamientos, en las dos dosis, estimularon uniformemente la actividad de la peroxidasa (POX) por encima del testigo, al final del ciclo (Cuadro 2). No obstante, los valores más altos se obtuvieron en los primeros tres muestreos a dosis completa y en la tercera fecha para las dos dosis, particularmente con fosetil-Al. En esta fecha también sobresalió la fluoxastrobina en dosis completa y metalaxil en media dosis, con valores muy cercanos a los del fosetil-Al. En el último muestreo la acción de la POX continuó descendiendo a valores casi imperceptibles en el testigo sin productos, mientras que los fungicidas la aumentaron ligeramente con respecto a su muestreo previo.

La POX oxida a los compuestos fenólicos y aumenta la velocidad de polimerización de éstos en sustancias similares a la lignina, que se depositan en las paredes en las plantas (Mclnnis et al., 2006). En este estudio, la acción se estimuló en la planta en ausencia de patógenos, principalmente a la mitad del ciclo por un fungicida multisitio (fosetil-Al), por un inhibidor de la respiración (fluoxastrobina) y por un inhibidor de la síntesis de ácidos nucleicos en hongos (metalaxil).

\section{Catalasa}

La actividad de la catalasa (CAT) disminuyó continuamente a lo largo del ciclo en las plantas testigo, mientras que los tratamientos estimularon su actividad mayormente a la mitad del ciclo con estímulo específico sobresaliente solo por fosetil-Al en media dosis. Las plantas testigo mostraron una acción mayor de la CAT que los tratamientos en el primer muestreo, pero posteriormente presentaron un descenso continuo de modo que su actividad final fue menor que cualquiera de los productos a dosis completa y similar a todos con medias dosis (Cuadro 2).

Aparentemente, las aplicaciones de medias dosis de los agroquímicos fueron imperceptibles para la planta durante la segunda mitad del ciclo, pues sus valores estuvieron cercanos a los del testigo, mientras que en dosis completa estimularon la acción de la CAT en el último muestreo con respecto al previo y al testigo. La CAT, al igual que la SOD, es una enzima antioxidante que cataliza la reducción-oxidación del peróxido de hidrógeno en agua y oxígeno, pero además se asocia con la muerte celular como respuesta de hipersensibilidad (Levine et al., 1994). Las acciones de las medias dosis también fueron imperceptibles en respuesta 
Cuadro 2. Comparación de medias $\left(\mathrm{U} \mathrm{g}^{-1}\right.$ peso fresco) entre los tratamientos, en cinco fechas de muestreo (M). DC, dosis completa; MD, media dosis. En negritas se marca el máximo valor alcanzado en cada fecha.

\begin{tabular}{|c|c|c|c|c|c|}
\hline Tratamientos & M 1 & M 2 & M 3 & M 4 & M 5 \\
\hline & & & Fenoles totales & & \\
\hline Metalaxil-m DC & $0.4093 d$ & $0.4520 \mathrm{bc}$ & $0.6253 a$ & $0.3883 a b$ & $0.3623 a b$ \\
\hline Metalaxil-m MD & $0.4013 d$ & $0.5360 a b$ & $0.5000 \mathrm{bcd}$ & $0.4453 a b$ & $0.3763 a b$ \\
\hline Fosetil-Al DC & $0.4040 \mathrm{~d}$ & $0.4930 \mathrm{abc}$ & $0.4347 d$ & $0.5053 a b$ & $0.3867 a b$ \\
\hline Fosetil-Al MD & $0.4530 \mathrm{c}$ & $0.5120 \mathrm{abc}$ & $0.6270 \mathrm{a}$ & $0.3523 b$ & $0.4607 \mathrm{~b}$ \\
\hline Mandiprop DC & $0.3996 d$ & $0.5223 a b$ & $0.5670 \mathrm{ab}$ & $0.3643 a b$ & $0.3713 a b$ \\
\hline Mandiprop MD & $0.4003 d$ & $0.4280 \mathrm{c}$ & $0.4397 \mathrm{~cd}$ & $0.4227 a b$ & $0.4047 a b$ \\
\hline Fluoxastrobin DC & $0.5020 a$ & $0.4590 \mathrm{bc}$ & $0.4743 \mathrm{bcd}$ & $0.5220 a$ & $0.5343 a$ \\
\hline Fluoxastrobin MD & $0.4820 \mathrm{~b}$ & $0.5790 \mathrm{a}$ & $0.5103 \mathrm{bcd}$ & $0.3970 a b$ & $0.4453 \mathrm{ab}$ \\
\hline \multirow[t]{2}{*}{ Testigo } & $0.4726 b$ & $0.5790 \mathrm{a}$ & $0.5327 \mathrm{abc}$ & $0.4603 a b$ & $0.4022 a b$ \\
\hline & \multicolumn{5}{|c|}{ Superóxido dismutasa } \\
\hline Metalaxil-m DC & $10.256 f$ & $42.420 \mathrm{c}$ & $38.370 \mathrm{c}$ & $43.060 \mathrm{a}$ & $4.836 \mathrm{~d}$ \\
\hline Metalaxil-m MD & $22.044 \mathrm{~b}$ & $94.580 \mathrm{a}$ & $46.710 \mathrm{bc}$ & $28.134 \mathrm{bcd}$ & 36.343 bc \\
\hline Fosetil-Al DC & 19.855 c & $46.710 \mathrm{c}$ & $84.060 \mathrm{a}$ & $14.191 \mathrm{bcd}$ & $30.952 \mathrm{a}$ \\
\hline Fosetil-Al MD & $15.402 d$ & $31.420 \mathrm{c}$ & $60.400 \mathrm{abc}$ & $23.370 \mathrm{bc}$ & $15.703 \mathrm{c}$ \\
\hline Mandiprop DC & $22.673 b$ & $50.130 \mathrm{bc}$ & $74.290 \mathrm{abc}$ & $25.400 \mathrm{bc}$ & $27.009 a b$ \\
\hline Mandiprop MD & $19.312 \mathrm{c}$ & $91.520 a b$ & $77.530 \mathrm{ab}$ & $13.643 \mathrm{~cd}$ & $13.622 \mathrm{~cd}$ \\
\hline Fluoxastrobin DC & $12.849 \mathrm{e}$ & $35.070 \mathrm{c}$ & $50.190 \mathrm{abc}$ & $7.812 \mathrm{~d}$ & $8.727 \mathrm{~cd}$ \\
\hline Fluoxastrobin MD & $25.136 \mathrm{a}$ & $92.680 \mathrm{a}$ & $84.370 \mathrm{a}$ & $28.134 b$ & $36.343 \mathrm{a}$ \\
\hline \multirow[t]{2}{*}{ Testigo } & $14.230 \mathrm{ed}$ & $93.400 \mathrm{a}$ & $41.440 \mathrm{c}$ & 17.034 bcd & $11.895 \mathrm{~cd}$ \\
\hline & & & Peroxidasa & & \\
\hline Metalaxil-m DC & $6869.300 \mathrm{~b}$ & $8888.400 a b$ & 8291.600 c & $5068.900 \mathrm{c}$ & 6022.200 c \\
\hline Metalaxil-m MD & $6828.400 \mathrm{~b}$ & 6624.000 ef & $9343.700 \mathrm{~b}$ & $5575.700 \mathrm{bc}$ & $6151.600 \mathrm{bc}$ \\
\hline Fosetil-Al DC & $8214.200 \mathrm{a}$ & $9219.800 \mathrm{a}$ & $9961.900 \mathrm{a}$ & $4344.800 \mathrm{~d}$ & $4946.000 \mathrm{~d}$ \\
\hline Fosetil-AI MD & $5582.400 \mathrm{c}$ & $5804.300 \mathrm{~g}$ & $9792.000 a b$ & 5555.700 bc & $5893.600 \mathrm{c}$ \\
\hline Mandiprop DC & $7002.700 \mathrm{~b}$ & $8866.600 a b$ & $7884.500 \mathrm{c}$ & $5766.100 \mathrm{~b}$ & $6846.300 \mathrm{a}$ \\
\hline Mandiprop MD & 6629.300 b & $7977.300 \mathrm{~cd}$ & 7869.000 c & $6675.900 \mathrm{a}$ & $3146.800 \mathrm{e}$ \\
\hline Fluoxastrobin DC & $3204.900 \mathrm{~d}$ & $6270.500 \mathrm{fg}$ & 9291.300 b & 5089.800 c & $6448.200 \mathrm{~b}$ \\
\hline Fluoxastrobin MD & $2836.400 \mathrm{~d}$ & 7288.900 de & $7721.500 \mathrm{~cd}$ & $5248.000 \mathrm{bc}$ & $4985.300 \mathrm{~d}$ \\
\hline \multirow[t]{2}{*}{ Testigo } & $5362.200 \mathrm{c}$ & 8342.100 bc & $7254.500 \mathrm{~d}$ & $3333.100 \mathrm{e}$ & $953.100 \mathrm{f}$ \\
\hline & & & Catalasa & & \\
\hline Metalaxil-m DC & $15,459 \mathrm{~cd}$ & $21,755 \mathrm{abc}$ & $22,204.9 \mathrm{dc}$ & $14,130 \mathrm{bc}$ & $16,037 \mathrm{ab}$ \\
\hline Metalaxil-m MD & $14,205 d$ & $16,757 \mathrm{c}$ & $26,350.7 \mathrm{ab}$ & 15,016 bc & $7046 \mathrm{~cd}$ \\
\hline Fosetil-Al DC & $25,273 b$ & $23,280 a b$ & $27,143.4 \mathrm{ab}$ & $11,625 \mathrm{dc}$ & $15,048 b$ \\
\hline Fosetil-Al MD & 22,368 bc & $27,343 a$ & $29,511.8 \mathrm{a}$ & $9034 d$ & $6353 \mathrm{~cd}$ \\
\hline Mandiprop DC & $26,170 \mathrm{~b}$ & $22,169 a b c$ & $21,080 d$ & $16557 a b$ & $19,240 \mathrm{a}$ \\
\hline Mandiprop MD & 21,886 bc & $21,712 \mathrm{abc}$ & $20,362.3 d$ & $19,124 a$ & $6353 d$ \\
\hline Fluoxastrobin DC & $17,464 \mathrm{~cd}$ & 17,931 bc & $25,410.6$ bc & $14,301 \mathrm{bc}$ & $15,291 b$ \\
\hline Fluoxastrobin MD & 19,686 bcd & 19,965 bc & $21,512.6 \mathrm{~d}$ & $16,380 a b$ & $6375 \mathrm{~cd}$ \\
\hline Testigo & $41,310 \mathrm{a}$ & $22,796 a b c$ & $19,456.2 d$ & $10,133 d$ & $8175 c$ \\
\hline
\end{tabular}

Medias dentro de un muestreo (columna) con las mismas letras son estadísticamente iguales (Tukey, 0.05). 
para la SOD, ya que los valores de este componente estuvieron cercanos a los del testigo.

\section{Correlaciones}

Las variables cuantificadas en el presente estudio corresponden a una ruta de metabolismo oxidativo, por lo que su respuesta podría estar, si no mutuamente dependiente, por lo menos relacionada. Cuando se aplicaron los productos en medias dosis, no se identificó correlación alguna con significancia estadística entre la presencia/actividad de las enzimas por efecto de metalaxil-m. No obstante, con estas mismas medias dosis se encontró coincidencia de comportamiento con significancia estadística entre las correlaciones POX/CAT por estimulación de mandipropamid, y de POX/SOD por fosetil-Al y fluoxastrobina (Cuadro 3).

Al aplicar dosis completas hubo correlación significativa de POX/CAT con metalaxil y fosetil-Al, al igual que de mandipropamid para FEN/SOD y fluoxastrobina para SOD/CAT. No obstante, con excepción de metalaxil, se correlacionaron entre ellas aunque de manera limitada y coincidente con fosetil-Al y fluoxastrobina, y diferente al testigo, que evidenció más su conexión de fenoles totales con SOD y POX. Con dosis completas las correlaciones se diversificaron; esto es, los cambios inducidos en cada una de las enzimas influyeron entre sí, y los productos en dosis completas actuaron y repercutieron en las plantas de manera dependiente para las diferentes enzimas. La respuesta de defensa implica la activación rápida de genes de transcripción que codifican un número de enzimas y no solo una (Manibhushanrao et al., 1988; Rudolph y Stahmann, 1964). El testigo (agua) presentó acción correlacionada únicamente entre FEN/SOD y FEN/POX.

\section{Consideraciones generales}

Al analizar semanalmente los máximos valores de respuesta enzimática inducida por los agroquímicos, con diferencia estadística significativa respecto al resto, resaltan dos de éstos: a) fosetil-Al $1.0 \mathrm{X}$, que indujo la mayor respuesta por POX durante las primeras tres semanas, y la mayor actividad de SOD en la tercera y quinta semana, y b) fluoxastrobina $0.5 \mathrm{X}$, de mayor estímulo para la actividad de SOD también durante las primeras tres semanas, y al igual que el fosetil-Al, también en la última (Cuadro 2, en negritas).

La ficha técnica del fosetil-Al lo menciona como de un modo de acción multisitio contra oomicetos, de movilidad bipolar y como estimulante de los "sistemas naturales de defensa de la planta" (http://www.agf.gov.bc.ca/pesticides/infosheets/fosetyl_al.pdf; Copping y Hewitt, 1998; Kuck et al., 2011), a lo que ahora se debe agregar que dicha estimulación es por lo menos para dos grandes grupos de enzimas en plantas, las peroxidasas y las superóxido dismutasas. Respecto a fluoxastrobina, solo se especifica que inhibe la respiración (http://www.farmchemicalsinternational.com/sourcing/research/ fluoxastrobin/; Copping y Hewitt, 1998; Kuck et al., 2011).

Los productos incluidos en el presente estudio estimularon respuestas comunes de defensa enzimática en ausencia de patógenos. En estudios similares se ha incluido la infección, tanto de virus como de un oomiceto (Juárez et al., 2010; Lozoya et al., 2006; Lozoya et al., 2007; Robledo et al., 2012). Con los resultados del presente estudio, y considerando la ausencia de patógenos, se puede afirmar que los estímulos y las diferencias con el testigo fueron por efecto de los agroquímicos exclusivamente. Así, a medida que se hicieron las aplicaciones de los productos se detectaron cambios en las enzimas incluidas en este estudio.

\begin{tabular}{|c|c|c|c|}
\hline & & Dosis & $r$ \\
\hline \multirow[t]{3}{*}{ POX-CAT } & Metalaxil & DC & 0.93 \\
\hline & Fosetil-Al & DC & 0.96 \\
\hline & Mandipropamida & MD & 0.94 \\
\hline \multirow[t]{2}{*}{ POX-SOD } & Fosetil & MD & 0.93 \\
\hline & Fluoxastrobina & MD & 0.89 \\
\hline \multirow[t]{2}{*}{ FEN-SOD } & Mandipropamida & DC & 0.95 \\
\hline & Testigo & & 0.89 \\
\hline CAT-SOD & Fluoxastrobina & DC & 0.91 \\
\hline FEN-POX & Testigo & & 0.97 \\
\hline
\end{tabular}

$\mathrm{DC}$, dosis completa; MD, media dosis. 
Destaca el estímulo inducido por fosetil-Al, fluoxastrobin y metalaxil-m, tanto en dosis media como en completa, aunque la hipótesis de que a mayor dosis de los productos correspondería mayor actividad enzimática no se cumplió, dado que a dosis media también se observó estímulo en algunas actividades enzimáticas.

\section{CONCLUSIONES}

La aplicación de fungicidas al follaje de papa en ausencia del patógeno estimuló la síntesis y acción de FEN, SOD, POX Y CAT. Las correlaciones entre las enzimas POX-CAT, POX-SOD, FEN-SOD Y FEN-POX con los agroquímicos fueron significativas, lo que indican similitud de respuestas o de dependencia entre ellas a más de un producto, de reacciones no específicas, por el estímulo químico externo. A lo largo del estudio se identificó especificidad temprana del fosetil-Al como estimulante de POX, y de la fluoxastrobina para SOD.

\section{BIBLIOGRAFÍA}

Agrios G. N. (2007) Fitopatología. 2a ed. Ed. LIMUSA, México. 819 p. Alia-Tejacal I., M. T. Colinas-León, M. T. Martínez- Damián y M. R. Soto-Hernández (2002) Factores fisiológicos, bioquímicos y de calidad en frutos de zapote mamey (Pouteria sapota Jacq) durante poscosecha. Revista Chapingo Serie Horticultura 8: 263-271.

Beltrán-García, M. J., T. Ogura-Fujii, G. Manzo-Sánchez y C. Arias-Castro (2006) Catalasas de hongos fitopatógenos: ¿Factores de virulencia y resistencia a fungicidas? Revista Mexicana de Fitopatología 24:50-58.

Beyer F. and W. Fridovich (1987) Assaying for superoxide dismutase activity: some large consequences of minor changes in conditions. Analytical Biochemistry 161:559-566.

Blackwell R. D., A. J. S. Murray and P. J. Lea (1990) Enzymes of photorespiratory carbon pathway. In: Methods in Plant Biochemistry. P. J. Lea (ed.). Academic Press, London, England. pp:129-144.

Bowler C., W. Van Camp, M. Van Montagu and D. Inze (1994) Superoxide dismutase in plants. Review of Plant Science 13:199-218.

Cahill D. M. and E. W. B. Ward (1989) Effects of metalaxyl on elicitor activity, simulation and glyceollin production and growth of sensitive and tolerant isolates of Phythophtora megasperma f. sp. Glycinea. Physiological and Molecular Plant Pathology 35:97-112.

Copping L. G. and H. G. Hewitt (1998) Chemistry and Mode of Action of Crop Protection Agents. The Royal Society of Chemistry, London, England. $164 \mathrm{p}$.

Cornide M. T., H. Lima y J. Surlí (1994) La Resistencia Genética de las Plantas Cultivadas. Ed. Científico- Técnica. La Habana, Cuba. $194 \mathrm{p}$.

Dai G. H., C. Andary, L. Mondolot-Cosson and D. Boubals (1995) Involvement of phenolic compounds in the resistance ofgrapevine callus to downy mildew (Plasmopara viticola). European Journal of Plant Pathology 101:541-547.

Deckers W. and L. Creasy (1989) Influence of fosetyl-Al on phytoalexin accumulation in Plasmopara viticola- grapevine interaction. Physiological and Molecular Plant Pathololy 34:203-213.

Flurkey W. H. and J. J. Jen (1978) Peroxidase and polyfenol oxidase activities in developing peaches. Journal of Food Sciences 43:1828-1831.

Gottsein H. D. and J. Kuúc (1989) Induction of systemic resistance to anthracnose in cucumber by phosphates. Phytopathology 79:176-179.

Hutcheson S. W. (1998) Current concepts of active defense in plants. Annual Review of Phytopathology 36:59-90.

Juárez C. A., H. Lozoya, M. T. Colinas y J. Bamberg (2010) Activación enzimática de especies de Solanum contra Phytophthora infestans. Interciencia 8:586-591.

Kessman H., T. Staub, C. Hofmann, T. Maetzke, G. Herzog, E. Ward, S. Uknes and J. Ryals (1994) Induction of systemic acquired disease resistance in plants by chemicals. Annual Review of Phytopathology 32:439-460

Kuck K., H., A. Leadbeater and U. Gisi (2011) FRAC Mode of Action Classification and Resistance Risk of Fungicides, in Modern Crop Protection Compounds, Volumes $1-3,2^{\text {nd }}$ ed. W. Krämer, $U$. Schirmer, P. Jeschke and M. Witschel (eds.). Wiley-VCH Verlag $\mathrm{GmbH} \&$ Co. KGaA, Weinheim, Germany. http://onlinelibrary. wiley.com/doi/10.1002/9783527644179.fmatter. (Noviembre 2015).

Kuúc J. (1995) Phytoalexins, Stress Metabolism, and Disease Resistance in Plants. Annual Review of Phytopathology 33:273-297.

Kuùc J. (1982) Induced immunity to plant disease. BioScience 32:854860

Levine A., R. Tenhhaaken, R. Dixon and C. Lamb (1994) $\mathrm{H}_{2} \mathrm{O}_{2}$ from the oxidative burst orchestrates the plant hypersensitive disease resistance response. Cell 79:583-593.

Lozoya-Saldaña H., G. Almanza-Serrano y M.T. Colinas-León (2006) Infección mixta Phytophthora infestans-Virus X de la papa (PVX) y mecanismos de defensa en papa (Solanum tuberosum L.) Agrociencia 40:753-764.

Lozoya-Saldaña H., R. Rivera-Hinojosa y M.T. Colinas-León (2007) Fenoles, peroxidasa y fenilalanina amonio liasa: Su relación con la resistencia genética de clones de papa (Solanum tuberosum L.) contra el tizón tardío (Phytophthora infestans Mont de Bary) Agrociencia 41:479-489.

Lück H. (1963) Catalase. In: Methods of Enzymatic Analysis. H. U. Bergmeyer (ed.) $2^{2}$ ed. Academic Press, N. Y. pp:885-888

Lyon G. D. and A. C. Newton (2000) Implementation of elicitor mediated induce resistance in agriculture. In: Induced Plant Defenses Against Pathogens and Herbivores: Biochemistry, Ecology and Agriculture. A. A. Agrawal, S. Tuzun, E. Bent (eds.). St. Paul, Minnesota, USA. APS Press. pp:299-318.

Manibhushanrao K., Z. Mohammed and N. Matsuyama (1988) Phenol metabolism and plant disease resistance. Acta Phytopathologica et Entomologica Hungarica 23:103-114

Martinez-Tellez M. A. and M. T. Lafuente (1997) Effect of high temperature conditioning of ethylene, phenylalanine ammonia-lyase and polyphenol oxidase activities in flavedoof chilled fortune marduly fruit. Journal of Plant Physiology 150:674-678.

McInnis S. M., D. C. Emery, R. Porter, R. Desikan, J. T. Hancock y S. J. Hiscock (2006) The role of stigma peroxidases in flowering plants insights from further characterization of a stigma-specific peroxidases (SSP) from Senecio squalidus (Asteraceae). Journal of Experimental Botany 8:1835-1846.

Monk L S., K. V. Fagerstedt and R. M. Crawford (1989) Oxygen toxicity and superoxide dismutase as antioxidant enzymes in physiological stress. Physiologia Plantarum 76:456-459.

Robledo E. M. N., H. Lozoya y M. T. Colinas (2012) Inducción de defensa en papa (Solanum tuberosum L.) por fungicidas. Interciencia 9:689-695.

Rudolph, K. and M. A. Stahmann (1964) Interaction of peroxidases and catalases between Phaseolus vulgaris and Pseudomonas phaseolicola. Nature 204:474-75.

Sigma-Aldrich Data sheet 47641 Folin-Ciocalteu's phenol reagent. https://www.sigmaaldrich.com/content/dam/sigma-aldrich/ docs/Sigma/Datasheet/6/47641 dat.pdf

Waterman, P. G. and S. Mole (1994) Analysis of Phenolic Plant Metabolites. Ed. Blackwell Scientific Publications. Oxford,UK. 238 p.

White R. F. (1979) Acetylsalicylic acid (aspirin) induces resistance to tobacco mosaic virus in tobacco. Virology 99:410-412. 